\title{
The Food and Drug Administration (FDA) and the European Medicines Agency (EMA) perspective on cardiovascular Polypill: A multidimensional concept
}

\author{
Mariusz Mogielnicki ${ }^{1 *}$, Damian Swieczkowski ${ }^{2 *}$, Witold Bachorski ${ }^{2}$, \\ Grzegorz Zuk ${ }^{2}$, Natasza Gilis-Malinowska ${ }^{2}$, Aleksander Zarzeka ${ }^{3}$, \\ Piotr Merks ${ }^{4}$, Marcin Gruchala ${ }^{2}$, Milosz Jaguszewski ${ }^{2}$ \\ ${ }^{1}$ Research and Development Laboratory, OCEANIC S.A., Poland \\ ${ }^{2} 1^{\text {st }}$ Department of Cardiology, Medical University of Gdansk, Poland \\ ${ }^{3}$ Division of Teaching and Learning Outcomes, Medical University of Warsaw, Poland \\ ${ }^{4}$ Department of Pharmaceutical Technology, Collegium Medicum in Bydgoszcz, \\ Nicolaus Copernicus University in Torun, Poland
}

\begin{abstract}
European Medicines Agency (EMA) and Food and Drug Administration (FDA) have the most powerful contribution to the development of law registration and efficient implementation of Polypill (and variety formulations with might be defined as fixed dose combination [FDC]) into clinical practice. Fixed-dose combinations are the consequence of evolution in pharmaceutical technology $[1,2]$. The first attempt of applying a FDC in human clinical settings has been observed since the 70 s. According to World Health Organization (WHO) experts, FDC in the secondary prevention of cardiovascular events are strongly recommended. Accordingly, FDA and EMA highlighted the potential benefits for the patient from the use of FDC regarding its clinical efficacy, low cost of therapy, and improving the adherence [3, 4]. Current European guidelines on cardiovascular diseases (CVD) prevention also recommend using FDC, due to patient convenience [5]. Currently, there is still no uniform and accepted definition of a Polypill. The early concept of Polypill was developed from combination of 5-6 active pharmaceutical ingredients (APIs) to formulation containing acetylsalicylic acid (ASA), statin and one antihypertensive agent (ramipril), which is the first Polypill approved in several Latin American and
\end{abstract}

European markets under the trade names Trinomia $^{\circledR}$, Sincronium ${ }^{\circledR}$, or Iltria ${ }^{\circledR}$ (Ferrer International, Spain) [6]. According to the Fuster-CNIC-Ferrer concept, Polypill is defined as an oral dosage form containing a low dose of ASA, statin, and at least one antihypertensive drug used in the prevention of cardiovascular events $[7,8]$.

According to WHO guidelines 2005 [9] for registration of FDC medicinal products, Polypill as FDC comprised of APIs of well-established use may be a subject of the three considered scenarios of the registration process, i.e. i) as a generic drug for existing FDC with established quality, safety, and efficacy; ii) as FDC of single entity products used together in a well-established therapeutic regime; iii) as FDC of APIs, which have not been previously used in combination to prevent CVD or a combination of well-established use, but in a different therapeutic regimen. Depending on the scenario, appropriate set of documentation in the registration process should be included; the most detailed documentation is required if Polypill is the subject of scenario III. In this case, an inclusion of rational justification of introducing it onto the market, estimating the benefits and risks of the proposed formulation and evaluation of safety and

Address for correspondence: Damian Swieczkowski, MPharm, Milosz J. Jaguszewski MD, PhD, FESC, $1^{\text {st }}$ Department of Cardiology, Medical University of Gdansk, ul. Dębinki 7, 80-211 Gdańsk, Poland, e-mail: d.swieczkowski@o2.pl; mjaguszewski@escardio.com.pl

*Equally contributed

Received: 10.09.2016 Accepted: 10.09.2016 
clinical efficacy in the registration dossier remains obligatory. However, in the case of scenario I and II, an applicant is obliged to perform the bioequivalence studies. Pre-clinical pharmacology and safety studies are not usually required in case of Polypill. Some of those requirements may be fulfilled by presenting relevant literature data [9].

In 2006, FDA issued guidance on registration of antiretroviral FDC, whereas there is no such a regulation for Polypills. The Agency is planning to release a general guide representing the FDA view on requirements for the registration process of FDC. According to FDA, a FDC is a formulation comprising at least two APIs available on the market as single entity products. An applicant is obliged to include the non-clinical safety assessment of the FDC in the registration dossier. Pharmacodynamic and toxicology additional non-clinical safety testing is not recommended before clinical trials for therapeutic regimens which are well known for safety of individual API and potential chemical interaction. In contrast, studies of potential toxicity to the developing embryo and fetus are recommended regardless of the knowledge of the toxic potential of APIs. Carcinogenicity study of FDC is recommended if such a potential was noticed during non-clinical studies. Bioequivalence studies are an obligation of a Marketing Authorization Holder (MAH) during the registration process of a generic drug. Once the data obtained from non-clinical studies do not suggest an interaction potential, testing on animals may be omitted before the clinical trials. In practice, FDA does not require clinical trials for registration of FDCs, which consist of APIs with well-established concomitant use. ASA-pravastatin and atorvastatin-amlodipine combinations were registered after establishing the lack of pharmacokinetic and pharmacodynamic interactions between the APIs [4].

According to the Directive 2001/83/EC of the European Parliament and of the Council of 6 November 2001 (OJ L 311, 28.11.2001, p. 67), the entity applying for registration of a medicinal product is not obliged to provide the results of toxicological and pharmacological tests or clinical trials when the components of the medicinal product have well-established medicinal use, with recognized efficacy and an acceptable level of safety based on literature data. In case of medicinal products registration containing known APIs which have not been used together in a given therapeutic indication, MAH is obliged to provide the results of toxicological and pharmacological tests, as well as clinical trials of the FDC. EMA guidelines of 2008 [10] govern non-clinical studies on the development of FDC medicinal products. In case of combined APIs of well-established use and with documented lack of interaction between the components, it is not required to conduct non-clinical studies. For well-established APIs, yet the undocumented use of their combination, the performance of an adequate non-clinical toxicology, pharmacokinetic and pharmacological studies is recommended [10].

Clinical studies during the development of FDC are also governed by the relevant guidelines (currently under revision). The joint use of Polypill components must have a rationale and provide tangible benefits in comparison with potential risks associated with the FDC. All of the APIs must have a proven action in the given indication, known safety profile and established pharmacokinetic and pharmacodynamic interactions. MAH can prove the action by presenting the results of clinical trials of Polypill and/or a combination of single entity products based on literature data or by comparing the results of clinical trials with literature data.

In case of the FDC containing at least three APIs such as Polypill, an applicant during the registration process must assess: i) potential for drug-drug interactions - pharmacokinetic studies in humans may be omitted if the appropriate in vitro test is conducted or a combination of APIs is of well-established use in medicine with specific safety profile or well-known pharmacokinetic and drug-drug interactions profile; ii) pharmacodynamic data - the entity is not obliged to carry out those tests, if present relevant clinical data concerning the efficacy and safety of used polytherapy; iii) the efficacy and safety of FDC, which must be higher than polypharmacy of single entity products; iv) bioequivalence of FDC to the single entity drugs according to EMA guidelines. In case of a new therapeutic regimen or different doses of Polypill in relation to single entity drugs, a similar pharmacokinetic profile and an evidence of the effectiveness of the proposed FDC must be well documented and strictly reported. In addition, an applicant should consider clinical safety issues. If the combination of APIs has new indications, the registration of the drug as an entirely new clinical development should be conducted [10].

The registration process of Polypill in both United States and European Union remains challenging and falls under many regulations related to FDC medicinal products. The FDA and the EMA requirements are similar and in case of Polypill consisting of APIs of well-established use under therapeutic regimen may be fulfilled mostly by 
literature. Indeed, the Polypill registration process may differ according to the law interpretation taken by the MAH and will be revised in the industrial regulatory practice.

\section{Conflict of interest: None declared}

\section{References}

1. de Cates AN, Farr MRB, Wright $\mathrm{N}$ et al. Fixed-dose combination therapy for the prevention of cardiovascular disease. Cochrane Database Syst Rev, 2014; 4: CD009868. doi: 10.1002/14651858. CD009868.pub2.

2. Khaled SA, Burley JC, Alexander MR, Yang J, Roberts CJ. 3D printing of tablets containing multiple drugs with defined release profiles. Int J Pharm, 2015; 494: 643-650. doi: 10.1016/j. ijpharm.2015.07.067.

3. European Medicines Agency Committee for Medicinal Products for Human Use. Guideline on the Investigation of Bioequivalence. [Internet]. 2010 [cited 18 January 2016]. Available from:http://www.ema.europa.eu/docs/en_GB/document_library/ /Scientific_guideline/2010/01/WC500070039.pdf. Accessed 18 Jan 2016.

4. Food and Drug Administration. FDA Briefing Document. Cardiovascular and Renal Drugs Advisory Committee Meeting To discuss the potential clinical utility of fixed- combination prescription drugs composed of an anti- hypertensive drug, aspirin, and a statin administered to reduce the risk of cardiovascular death, nonfatal myocardial infarction, and nonfatal stroke in patients with a history of cardiovascular disease. [Internet]. 2014 [cited 7 January 2016]. Available from: http://www.fda.gov/downloads/
/AdvisoryCommittees/CommitteesMeetingMaterials/Drugs/CardiovascularandRenalDrugsAdvisoryCommittee/UCM413061.pdf.

5. Piepoli MF, Hoes AW, Agewall S et al. 2016 European Guidelines on cardiovascular disease prevention in clinical practice: The Sixth Joint Task Force of the European Society of Cardiology and Other Societies on Cardiovascular Disease Prevention in Clinical Practice (constituted by representatives of 10 societies and by invited experts): Developed with the special contribution of the European Association for Cardiovascular Prevention \& Rehabilitation (EACPR). Eur Heart J, 2016. doi: 10.1093/ /eurheartj/ehw106.

6. Tamargo J, Castellano JM, Fuster V. The Fuster-CNIC-Ferrer Cardiovascular Polypill: A polypill for secondary cardiovascular prevention. Int J Cardiol, 2015; 201: S15-S22.

7. Castellano JM, Bueno H, Fuster V. The cardiovascular polypill: Clinical data and ongoing studies. Int J Cardiol, 2015: S8-S14. doi: 10.1016/S0167-5273(15)31027-5.

8. Selak V, Harwood M, Raina Elley C et al. Polypill-based therapy likely to reduce ethnic inequities in use of cardiovascular preventive medications: Findings from a pragmatic randomised controlled trial. Eur J Prev Cardiol, 2016; 23: 1537-1545. doi: 10.1177/2047487316637196.

9. World Health Organization. Guidelines for registration of fixeddose combination medicinal products. WHO Technical Report Series, 2005; 929: 94-142. [Internet]. 2005 [cited 7 January 2016]. Available from: http://apps.who.int/prequal/info_general/ /documents/trs929/who_trs_929.pdf. Accesed 7 Jan 2016.

10. European Medicines Agency Committee for Human Medicinal Products. Guideline on clinical development of fixed combination medicinal products. 2015. http://www.ema.europa.eu/ /docs/en_GB/document_library/Scientific_guideline/2015/05/ /WC500186840.pdf. Accessed 3 Feb 2016. 